\title{
The effect of size and composition on structural transitions in monometallic nanoparticles ${ }^{\star}$
}

\author{
Kevin Rossi ${ }^{1, a}$, Luca Pavan $^{1}$, YeeYeen Soon ${ }^{2}$, and Francesca Baletto ${ }^{1}$ \\ ${ }^{1}$ King's College, Physics Department, Strand WC2R 2LS, London, UK \\ ${ }^{2}$ Universiti Saints, School of Physics, 11800 USM, Penang, Malaysia
}

Received 18 May 2017 / Received in final form 10 July 2017

Published online 12 February 2018

(C) The Author(s) 2018. This article is published with open access at Springerlink.com

\begin{abstract}
Predicting the morphological stability of nanoparticles is an essential step towards the accurate modelling of their chemophysical properties. Here we investigate solid-solid transitions in monometallic clusters of $0.5-2.0 \mathrm{~nm}$ diameter at finite temperatures and we report the complex dependence of the rearrangement mechanism on the nanoparticle's composition and size. The concerted Lipscomb's DiamondSquare-Diamond mechanisms which connects the decahedral or the cuboctahedral to the icosahedral basins, take place only below a material dependent critical size above which surface diffusion prevails and leads to low-symmetry and defected shapes still belonging to the initial basin.
\end{abstract}

\section{Introduction}

Morphology, together with size and composition, determines nanocluster's chemophysical properties, establishing a profound link between structural stability and reliable performance [1-4]. One of the most clear example is in nanocatalysts, as the adsorption properties depend on the local environment of each site, with less coordinated atoms binding small molecules more strongly. Changes in the nanoparticle morphology affect the site coordination, hence altering cluster reactivity and selectivity with respect to a given reaction $[2,3]$. Predicting the extent by which structural fluctuations take place is then a fundamental step in order to quantify and possibly tune their chemophysical properties.

Structural rearrangements have been detected at single atom precision [5-7] and protocols to trigger them by means of annealing or electron beam irradiation have been developed, greatly advancing the control and understanding of such processes [8-10]. Numerical modelling can complement and aid experimental microscopy. They allow for full atomistic detail of the system and enable to single out different parameters (e.g. temperature, size, composition) to clarify the driving mechanisms of the kinetic processes of interest. Following geometrical intuition, the Diamond-Square-Diamond (DSD) mechanisms, can always connect perfect close-shell cuboctahedra (Co) and Ino-decahedra (Dh) to the icosahedra (Ih), via a concerted screw dislocation motion, regardless of cluster size and atomic type [11-13]. It has been demonstrated

\footnotetext{
* Contribution to the Topical Issue "Shaping Nanocatalysts", edited by Francesca Baletto, Roy L. Johnston, Jochen Blumberger and Alex Shluger.

${ }^{a}$ e-mail: kevin.rossi@kcl.ac.uk
}

that $\mathrm{Dh} \rightarrow \mathrm{Ih}$ and $\mathrm{Co} \rightarrow \mathrm{Ih}$ transitions paths take place in monometallic clusters up to 147 atoms via the DSD [1420]. They can be hindered in nanoalloys with unfavourable chemical ordering or large mismatch $[21,22]$, and in the case of supported clusters [23].

The energetic cost of concerted rearrangements is generally expected to be significantly lower than multi-step mechanisms [24]. Activation energy barriers for correlated movements are expected to increase with the number of components involved in the process [25-27], yet previous studies did not systematically address whether DSD mechanisms are accessible at finite temperatures and are energetically competitive with respect to others, regardless of cluster nuclearity and material. Here, we report on structural transitions in noble and quasi-noble metallic nanoparticles with a diameter in the $0.5-2.0 \mathrm{~nm}$ range at room temperature, and during a melting process. Our results show how size-effects not only influence thermal properties but also what reshaping pathways can be thermally activated. We identify fascinating size and material dependencies of the shape transformations: DSD mechanisms, connecting different structural basins, result hindered or suppressed above a certain size where surface diffusion becomes predominant. The latter leads to highly defected structures within the same morphological basin, i.e. the original number of 5 -fold axis in the structure is preserved.

\section{Models and methods}

Clusters made of face centered cubic (FCC) metals considered - like $\mathrm{Cu}, \mathrm{Ag}, \mathrm{Au}, \mathrm{Ni}, \mathrm{Pd}, \mathrm{Pt}$ - commonly display three main morphologies at the nanoscale: (i) FCC-cuts (e.g. Co); (ii) multiple-twins shapes with a single 5 -fold 


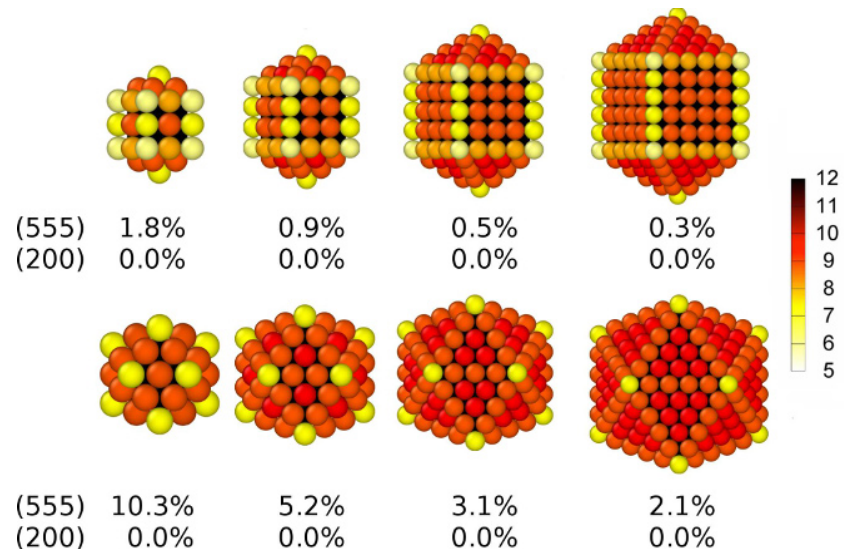

Fig. 1. Closed-shell Dh (top) and Ih (bottom) clusters of $55,147,309,561$ atoms. Atoms are coloured according to their coordination, defined as the number of neighbours falling within a cut-off distance of $\sqrt{2} / 2$ times the metal bulk lattice parameter. The percentage of (555) and (200) CNA signature is given as reference.

symmetry axis (e.g. Dh); (iii) highly symmetric geometries with six 5 -fold axes (e.g. Ih). Generally speaking, Ih are favourable at very small sizes, FCC structures at very large approaching the bulk limit and $\mathrm{Dh}$ in between. The crossover sizes can be recast in term of the inter-particle potential stickiness, with soft potentials showing a strong tendency to form twinned nanoparticles over a wider size range [28]. Focusing on clusters with a diameter of $0.5-$ $2.0 \mathrm{~nm}$, we study solid-solid transitions connecting the Ih and Dh geometrical basin at magic closed shell sizes - 55, 147, 309, 561 - as shown in Figure 1. Similar conclusions can be obtained for the rearrangements connecting the Co and Ih basins as reported in Appendix A.

At the basis of the characterisation of solid-solid transitions we need a measure to identify when the nanoparticle explores a different morphological basin or when it remains in the starting one, even if defects and distortions are present. In the considered systems we expect a variation of the number of 5 -fold axis for mechanisms connecting different structural basins. Structural rearrangements within the same morphological basin are instead mainly characterised by surface reconstructions leading the formation of defects, islands, steps, and re-entrances.

Common Neighbour Analysis (CNA) provides a robust way to detect changes of the cluster geometry [29]. CNA assigns a signature $(r, s, t)$ to each pair of nearest neighbours depending on the number of common neighbour atoms they share $(r)$, the total number of bonds among the $r$ atoms $(s)$, and the length of the largest chain formed by the $s$ bonds $(t)$. We monitor the percentage of pairs presenting a $(5,5,5)$ signature, corresponding to pairs lying on a 5 -fold axis, and the percentage of $(2,0,0)$ ones, signature which characterizes pairs belonging to a $(1,1,0)$ facet. Notwithstanding CNA signatures accurately detail changes in the cluster architecture, they do not explicitly encode information on the transition mechanism.
Solid-solid transitions can be considered rare events. To detect them we adopt and compare two synergic methodologies based upon molecular dynamics (MD): metadynamics (MetaD) and iterative temperature molecular dynamics (itMD) as implemented in the LoDiS package. Equations of motion are solved using the velocity-Verlet algorithm with a time step of $5 \mathrm{fs}$, and the temperature of the system is regulated by an Andersen thermostat, where the frequency is set in order not to alter diffusion properties. Metal-metal interactions are modelled within the second moment approximation of the tight binding theory [30], following parametrisations available in literature [28].

MetaD enhances the sampling of solid-solid transitions at a fixed temperature by adding a history dependent meta-potential built in a reduced dimensionality collective variables (CV) space [31]. We recently proposed a set of CV to faithfully drive and coarse the dynamics of the nanoparticles, and probe structural transitions between closed-shell polyhedra nanoclusters by promoting vibrational breathing-related modes and concerted sliding and rotations along (111) planes [20]. It consists of two window functions (WF), positioned at characteristic distances, $d_{0}$, of the pair distance distribution functions:

$$
\mathrm{WF}=\sum_{i, j ; i \neq j} \frac{1-\left(\frac{r_{i j}-d_{0}}{r_{0}}\right)^{6}}{1-\left(\frac{r_{i j}-d_{0}}{r_{0}}\right)^{12}},
$$

where $r_{i j}$ is the distance between $i$ and $j$ atoms, and $r_{0}$ the window width. The first WF is set at a hexagonal close packed crystals peak $\left(d_{0} \sim \sqrt{8} / 3\right.$ times the metal bulk lattice parameter) to enumerate the stacking fault number, the second one is centred at the 5th nearest neighbours distance. The pair distance distribution function of Ih, Dh and Co structures, indeed, present different profiles at these characteristic distances. For both WF, we set a width $r_{0}$ equal to the five percent of the bulk lattice parameter. The MetaD potential evolves every $10 \mathrm{ps}$ by the deposition of a Gaussian of height $0.05 \mathrm{eV}$ times the number of shells in the clusters ( 1 for 55 atoms, 2 for $147, \ldots$ ) and a width equals to 15 times the number of cluster shells. When two different morphological basins are explored, e.g. following the subsequent $\mathrm{Dh} \rightarrow \mathrm{Ih}$ and $\mathrm{Ih} \rightarrow$ Dh transitions, the free energy barriers dividing them, $\Delta F_{\rightarrow I h}$ and $\Delta F_{\rightarrow D h}$, can be reconstructed as the negative of the meta-potential. Due to the roughness of the energy landscapes several different high energy minima are commonly observed after the transition between the closed-shell polyhedra of interest. We report $\overline{\Delta F_{\rightarrow I h}}$, the mean value of $\Delta F_{\rightarrow I h}$, obtained by averaging over at least eight independent MetaD runs at a temperature of $300 \mathrm{~K}$. The error is calculated as the absolute largest difference between $\overline{\Delta F_{\rightarrow I h}}$ and the $\Delta F_{\rightarrow I h}$ found from each single independent run. When the initial Dh basin is left and not explored any more during the course of the MetaD, we estimate the free energy barrier, $\overline{\Delta F_{\rightarrow I h}^{*}}$, as the highest point in the reconstructed energy landscape. This is an heuristic measure and it represents an upper bound on the true free energy barrier. 
ItMD consists of several concatenated MD runs where the temperature is slowly raised at a constant rate of $10 \mathrm{~K}$ every $1 \mathrm{~ns}$. In between two subsequent increments, the system is free to evolve according to Newton's equation of motion. This time scale is longer than the typical prefactor for the single adatom diffusion on extended metallic surfaces and allows us to monitor the appearance of any structural instabilities before the melting transition. A focus is set to determine the temperature at which the cluster changes into an Ih, $T_{\rightarrow I h}$ or to a defected Ino- or Marks-Decahedron, characterized by at least two atoms change their coordination to $10, \mathrm{~T}_{\rightarrow d D h}$. In Table 1 we report the average from the eight independent itMD runs of $T_{\rightarrow I h}$ and $\mathrm{T}_{\rightarrow d D h}, \overline{T_{\rightarrow I h}}$ and $\overline{T_{\rightarrow d D h}}$. To allow for an easy comparison of systems with different composition and size the reported temperatures are normalized with respect to the melting temperature of the Ih for that size and composition. The melting transition is identified by visual inspection of the caloric curve (potential energy versus temperature) which displays a sharp increase in correspondence of the phase transition. The error on $\overline{T_{\rightarrow I h}}$ and $\overline{T_{\rightarrow d D h}}$ is calculated as the largest absolute deviation of $T_{\rightarrow I h}$ and $T_{\rightarrow d D h}$ for each independent run and their mean value.

To characterize the rearrangement mechanisms during each dynamic, we monitor the number of changes in the first coordination shell of each atom $i, R_{i}$, by contrasting the nearest neighbour adjacency matrix of the system, $M_{i j}(t)$ at time $t$ and $t+\Delta t$ :

$$
R_{i}(t, t+\Delta t)=\sum_{j \neq i}^{N}\left|M_{i j}(t+\Delta t)-M_{i j}(t)\right|,
$$

where $N$ is the total number of atoms in the cluster. $M_{i j}(t)$ is a $N$ by $N$ matrix with entries equal to $1(0)$ when atoms $i$ and $j$ are (not) nearest neighbours. Two atoms are defined nearest neighbours if their distance is less than the one where the system pair distance distribution function presents its first minimum. No changes in the $i$ th neighbours lead to $R_{i}(t, t+\Delta t)=0$, while the maximum change for a FCC bulk atom is $R_{i}(t, t+\Delta t)=24$, twice the number of its nearest neighbours.

To estimate the collectivity of a mechanism, $H$, we count the portion of atoms changing at least one nearest neighbour, during $\Delta t$ time, and the total number of atoms in the cluster:

$H(t, t+\Delta t)=\sum_{i}^{N} \frac{\Theta\left(R_{i}(t, t+\Delta t)\right)}{N}\left\{\begin{array}{l}\Theta(0)=0 \\ \Theta\left(R_{i}\right)=1 \forall R_{i}>0\end{array}\right.$

$H$ ranges between 0 and 1: $H=0$ when no bond has been broken or formed, $H=1$ when all atoms change at least one neighbour.

Furthermore, we define the degree by which a rearrangement is concerted, $C$, as the difference between the number of atoms involved in the process between $t-\Delta t$ and $t$, and between $t$ and $t+\Delta t$.

$$
C(t-\Delta t, t, t+\Delta t)=|H(t-\Delta t, t)-H(t, t+\Delta t)| .
$$

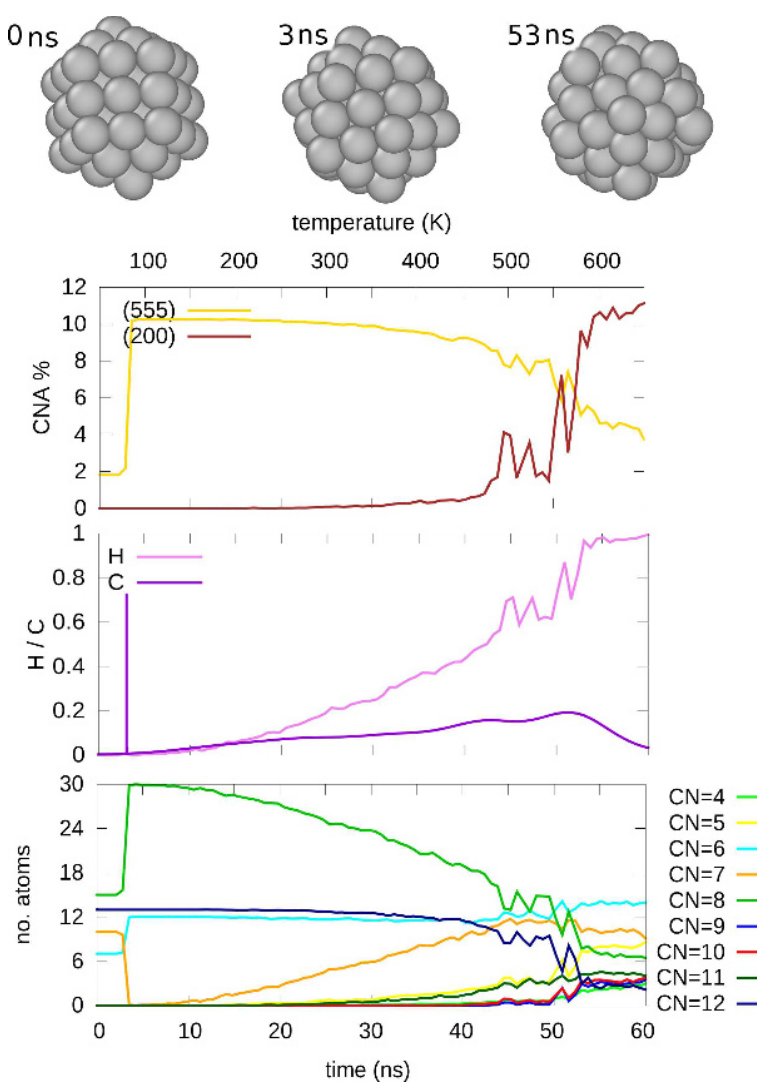

Fig. 2. Structure and rearrangement characterization for a Dh to Ih transition in Ag55, sampled by itMD. The top panel displays snapshots of representative structures observed during the simulation and the corresponding time at which they are first formed. Lower panels show the evolution of the (555) and (200) CNA signature percentages, $H$ and $C$ indexes, and coordination number distributions during the course of the simulation.

If all atoms undergo a change in their neighbourhood at the time $t$, but none in the successive one $C(t-$ $\Delta t, t, t+\Delta t)$ peaks at its maximum value of 1 . Single-step mechanisms involving only a sector of the cluster, or multistep processes are characterized by lower values of $C(t-$ $\Delta t, t, t+\Delta t)$, while continuous atomic rearrangements display $C \sim 0$.

$H$ and $C$ depend on the choice of $\Delta t$, which has to capture the fastest structural movement of interest. A too large $\Delta t$ may lead to a dramatic change in the $H$ and $C$ estimate by coarsening different atomic rearrangements into a single instance. On the other hand, a too small $\Delta t$ may disguise a single step rearrangement as a multistep one because, although the rearrangement of several atoms neighbours is simultaneous, the degree in the bond breaking or elongation might not be identical for each pair, leading to an artefact discretisation of how the system adjacency matrix varies. In the following, we opt for $\Delta t=$ $10 \mathrm{ps}$, roughly the time scale over of diffusion process on low Miller index facets, as it represents the fastest process we want to discriminate.

Of particular interest in our investigation is the determination of the highest value of $\mathrm{C}$ observed during the 
Table 1. Per each system (leftmost column) we report $\rightarrow \operatorname{Ih} \%, \overline{C_{\rightarrow I h}^{\max }}$ and $\overline{T_{\rightarrow I h}}$, or, $\overline{C_{T_{\rightarrow d D h}}^{\max }}$ and $\overline{T_{\rightarrow d D h}}$, for simulations ending respectively in the Ih or defected-Dh $(\mathrm{dDh})$ funnel as found via itMD. The error on $\overline{C_{\rightarrow I h}^{\max }}, \overline{C_{\rightarrow d D H}^{\max }}$ is around 0.1 for the concertedness index and of 0.03 for the characteristic transition temperatures $\overline{T_{\rightarrow I h}}$, and $\overline{T_{\rightarrow d D h}}$. Rightmost column reports $\overline{\Delta F_{\rightarrow I h}}$, and $\overline{\Delta F_{\rightarrow D h}}$ in bracket if observed. Error on $\overline{\Delta F_{\rightarrow I h}}\left(\overline{\Delta F_{\rightarrow D h}}\right)$ is of the order of the height of one Gaussian. $\overline{\Delta F_{\rightarrow I h}^{*}}$ instead represents an upper bound of the true free energy barrier.

\begin{tabular}{|c|c|c|c|c|c|c|c|}
\hline System & $\rightarrow \operatorname{Ih} \%$ & $\overline{\overline{C_{\rightarrow I h}^{m a x}}}$ & $\overline{T_{\rightarrow I h}}$ & $\overline{\overline{C_{\rightarrow d D h}^{\max }}}$ & $\overline{\overline{T_{\rightarrow d D h}}}$ & $\overline{\Delta F_{\rightarrow I h}^{(*)}}\left(\overline{\Delta F_{\rightarrow D h}}\right)$ & $(\mathrm{eV})$ \\
\hline $\mathrm{Ni}_{55}$ & 100 & 0.9 & $<0.02$ & & & $0.05(1.25)$ & \\
\hline $\mathrm{Ni}_{147}$ & 100 & 0.7 & 0.37 & & & 0.2 & \\
\hline $\mathrm{Ni}_{309}$ & 38 & 0.2 & 0.85 & 0.3 & 0.37 & 0.6 & \\
\hline $\mathrm{Ni}_{561}$ & 0 & & & 0.2 & 0.33 & 1.3 & \\
\hline $\mathrm{Pd}_{55}$ & 100 & 0.7 & 0.20 & & & $0.05(1.4)$ & \\
\hline $\mathrm{Pd}_{147}$ & 100 & 0.7 & 0.55 & & & 0.9 & \\
\hline $\mathrm{Pd}_{309}$ & 0 & & & 0.2 & 0.57 & 3.8 & \\
\hline $\mathrm{Pd}_{561}$ & 0 & & & 0.2 & 0.52 & & \\
\hline $\mathrm{Pt}_{55}$ & 100 & 0.7 & 0.36 & & & $0.3(1.3)$ & \\
\hline $\mathrm{Pt}_{147}$ & 100 & 0.7 & 0.58 & & & 1.9 & \\
\hline $\mathrm{Pt}_{309}$ & 0 & & & 0.2 & 0.57 & 7.4 & \\
\hline $\mathrm{Pt}_{561}$ & 0 & & & 0.2 & 0.45 & & \\
\hline $\mathrm{Cu}_{55}$ & 100 & 1.0 & $<0.02$ & & & $<0.05$ & \\
\hline $\mathrm{Cu}_{147}$ & 100 & 1.0 & $<0.02$ & & & 0.2 & \\
\hline $\mathrm{Cu}_{309}$ & 100 & 0.6 & 0.24 & & & 0.6 & \\
\hline $\mathrm{Cu}_{561}$ & 100 & 0.4 & 0.61 & & & 1.3 & \\
\hline $\mathrm{Ag}_{55}$ & 100 & 0.7 & 0.19 & & & $0.05(1.4)$ & \\
\hline $\mathrm{Ag}_{147}$ & 100 & 0.6 & 0.51 & & & $0.6(4.3)$ & \\
\hline $\mathrm{Ag}_{309}$ & 0 & & & 0.2 & 0.55 & 3.7 & \\
\hline $\mathrm{Ag}_{561}$ & 0 & & & 0.2 & 0.50 & & \\
\hline $\mathrm{Au}_{55}$ & 100 & 0.7 & 0.43 & & & $0.05(0.9)$ & \\
\hline $\mathrm{Au}_{147}$ & 0 & & & 0.4 & 0.52 & 0.5 & \\
\hline $\mathrm{Au}_{309}$ & 0 & & & 0.2 & 0.48 & & \\
\hline $\mathrm{Au}_{561}$ & 0 & & & 0.1 & 0.42 & & \\
\hline
\end{tabular}

structural rearrangement towards the $\mathrm{Ih}$ or dDh basin, respectively identified as $C_{\rightarrow I h}^{\max }$ and $C_{T \rightarrow d D h}^{\max }$. After estimating them during each independent itMD run, we report their average $\overline{C_{\rightarrow I h}^{\max }}$ and $\overline{C_{T \rightarrow \text { max }}^{\max }}$. The error associated their measure is estimated as described for the case of $\overline{T_{\rightarrow I h}}, \overline{T_{\rightarrow d D h}}, \overline{\Delta F_{\rightarrow I h}}$, and $\overline{\Delta F_{\rightarrow D h}}$.

\section{Results and discussion}

\subsection{Architecture and rearrangement characterization}

The cluster dynamical evolution from a Dh is summarised in Table 1, where we report for each chemical species and cluster: the proportion of runs sampling Ih before melting, $\rightarrow I h \%$, the transition temperature towards the $\mathrm{Ih}$ or $\mathrm{dDh}$ basin, $\overline{T_{\rightarrow I h}}$ or $\overline{T_{\rightarrow d D h}}$, and the mean highest $C$ value for dynamics ending in either of the two basins, $\overline{C_{\rightarrow I h}^{\max }}$ or $\overline{C_{\rightarrow d D h}^{\max }}$, the free energy barrier for the direct $\mathrm{Dh} \rightarrow \mathrm{Ih}$ (Ih $\rightarrow \mathrm{Dh}$ ) transition, $\overline{\Delta F_{\rightarrow I h}^{*}}\left(\overline{\Delta F_{\rightarrow D h}}\right.$, if observed).

We note that under the itMD scheme all clusters either transform into an Ih or remain trapped in a decahedral morphology, even if defected. We further observe that concerted motions consistently drive the system out of the original geometrical basin, while surface adatom diffusion constrains shape fluctuations to the original structure morphological basin. $\overline{T_{\rightarrow I h}}$ and $\overline{\Delta F_{\rightarrow I h}}$ increase while $\overline{T \rightarrow D h}$ decreases with cluster size: concerted mechanisms in large clusters involve more atoms, thus result increasingly expensive, while the energetic cost for adatom hopping or exchange, driving the formation of surface defect is independent of cluster size.

Solid-solid transitions towards the Ih via the DSD mechanisms are systematically observed in all nanoparticles of 55 atoms regardless of their chemical composition. A paradigmatic example of the transition for $\mathrm{Ag}_{55}$ is shown in Figure 2: the rearrangement takes place via a single step and the intermediate configuration between the perfect geometries displays the diamond facets characteristic of the Diamond-Square-Diamond mechanisms. CNA (555)\% sharply moves from 1.8 to 10.3 , while the (200)\% is constant at 0 . The simultaneous and concerted rearrangement leads to a peaked value of the $H$ and $C$ parameters in correspondence of the transition. The martensitic character of the transformation is highlighted by the evolution of the coordination number distributions where no atom has 


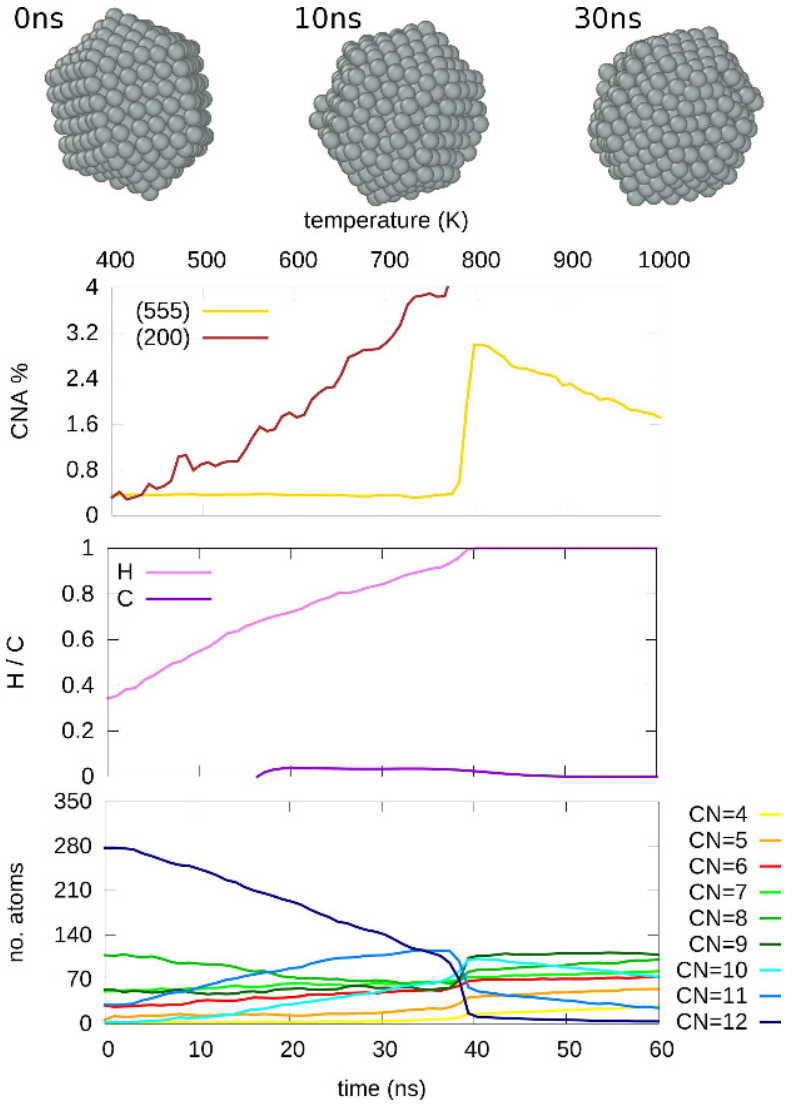

Fig. 3. Structure and rearrangement characterization for a $\mathrm{Dh}$ to defected Dh transition in Ag561, sampled by itMD. Panel ordering follows what described in Figure 2.

coordination 11, 10 or less then 4 . After the Ih formation, edge atoms are quite mobile and their coordination fluctuates between 7 and 8 , with a decrement of the population of seven coordinated atoms with higher temperatures, not due to the formation of a (100) facet, but because of the broadening of the first and second nearest neighbour shells. We highlight the nucleation of defects on the surface, just before the melting transition. Rosette defects, as the one evident in the snapshot taken at 53 ns (Fig. 2), are commonly observed at high $\mathrm{T}$, suggesting they are entropically favourable. MetaD runs for 55 atoms clusters sample diffusively the $\mathrm{Ih}$ and $\mathrm{Dh}$ basin before exploring a series of low-symmetry structures including twinned FCC. The $\overline{\Delta F_{\rightarrow I h}}\left(\overline{\Delta F_{\rightarrow D h}}\right)$ for transitions in 55 atoms clusters is in the order of the $0.15(1.25) \mathrm{eV}$, regardless of cluster composition, in agreement with previous results [13].

DSD rearrangements towards the Ih basin are observed in all the 147 atoms systems, apart for Au. During the transition the (555) CNA signature percentage shifts to the Ih ideal value while no defects are observed both when looking at the (200) signature occurrence and the coordination number distributions. By the same token $H$ and $C$ display a spike towards values close to 1 when the transition takes place. The energetic cost for rearrangements towards the icosahedral basin $(\sim 0.15 \mathrm{eV}$ for 55 atoms clusters, $0.5-1.5 \mathrm{eV}$ for 147 ones) is similar to the one for adatom diffusion via hopping on an homoatomic (100) surface (0.37-0.88 eV [27]), with the former transition leading, generally, to much more energetically favourable structures. Also in the case of 147 atoms, defects in the surface of the Ih arise before the melting transition.

We mention that $\mathrm{Au}_{147}$ displays only $\mathrm{Dh} \rightarrow$ defected $\mathrm{Dh}$ transition under the itMD scheme. The transition comprises surface defects formation, leading to an increase in the number of low-coordinated atoms and (200) CNA signature pairs. $H$ increases with temperature, yet, $C$ does not display any net trend, with spikes to a maximum value of 0.2 before the melting transition where it drops to 0 . The 5 -fold axis remains of the same length while the $\mathrm{Dh}$ structure changes into a Marks-Dh: the initial (100) square facets are disrupted while (110) re-entrances are formed. For MetaD simulations, $\mathrm{Au}_{147} \mathrm{Dh}$ still shows the transformation towards a defected Ih shape after the formation of few surface defects. The transition takes place via a DSD rearrangement in the inner shells of the clusters which propagates to the defected surface, as revealed by the peak around 0.4 of the $C$ index when the transformation takes place. The defected Ih structure commonly display the formation of a 6-coordinated ring around a vertex [32], together with few adatoms diffusing on the facets, thus the (555) signature percentage is slightly lower than the one for a closed-shell Ih while the number of 10 or 11 coordinated atoms is not null.

Martensitic transitions towards the Ih can not be sampled in 309 and 561 atoms nanoparticles via itMD, apart for $\mathrm{Ni}_{309}, \mathrm{Cu}_{309}$ and $\mathrm{Cu}_{561}$, in agreement with what observed in previous studies employing similar heating rates $[17,18]$. Dh reorganizes into a structure presenting defects and re-entrances formed by adatom hopping and exchanges from edges and corner to facets. Structure and kinetics characterization for a selected itMD simulation is reported in Figure 3 where we analyze the case of a $\mathrm{Ag}_{561}$ Dh transition towards a defected Marks-Dh. The high mobility of the cluster surface during the rearrangements corresponds to a continuous and non monotonous change in the CNA signatures and coordination number population distributions. While $H$ shows an overall increasing trend with temperature, $C$ always displays relatively low values, corresponding to a non-concerted rearrangement. The $(5,5,5)$ CNA signature percentage signals that the clusters not only preserve the number of 5 -fold axis in the structure but also all the atoms along it. Defects can be appreciated in greater numbers for higher temperatures, corresponding to an increase in the $(2,0,0)$ signature percentage, low coordinated atoms, and atomic mobility. This behaviour is expected from an entropic argument and in relation to the probability for adatom diffusion on a low-Miller index surface at high temperature.

$\mathrm{Cu}_{309}$ and $\mathrm{Cu}_{561}$ display martensitic $\mathrm{Dh} \rightarrow \mathrm{Ih}$ transitions both during itMD and MetaD sampling. The rearrangement takes place via a pathway similar to the DSD one, yet in a layer-by-layer fashion. The two steps transition is characterized by an intermediate configuration presenting a mix of (100) and (111) facets, as witnessed by the presence of both 6 and 5 coordinated vertexes, and a number of (555) CNA signature in between 
the ideal Dh and Ih ones. $H$ and $C$ present a value close to 0.5 in correspondence of the two partial rearrangements. $\mathrm{Ni}_{309}$ rearrangements from the Dh structure towards the Ih basin instead happen in a similar fashion to the $\mathrm{Dh} \rightarrow \mathrm{Ih}$ transition observed in $\mathrm{Au}_{147}$ by means of MetaD. The formation of surface defects is followed by a inner shells DSD driving the system towards in the Ih funnel while partial surface melting is observed. According to MetaD, notwithstanding the competition with surface peeling and defects formation, rearrangements to and from the Ih basin for 309 and 561 atoms clusters via layer-by-layer DSD mechanism result accessible not only for $\mathrm{Cu}$ but also for $\mathrm{Ni}$, $\mathrm{Pd}, \mathrm{Pt}$, and $\mathrm{Ag}$.

We would like to highlight the material-dependence of the size range where concerted mechanisms are likely to happen. They result already hindered, if not forbidden, in Au clusters larger than 147 atoms, Pt clusters above 309 atoms and $\mathrm{Ni}, \mathrm{Ag}$, and $\mathrm{Pd}$ clusters with a nuclearity higher than 561 atoms. In the case of $\mathrm{Cu}$, the crossover is expected above the sizes here considered, as both at 309 and 561 a layer-by-layer DSD is observed. Similarly, Au and $\mathrm{Pt}$ favour Marks-like re-entrances already for clusters as small as 100 atoms, while $\mathrm{Ag}, \mathrm{Ni}$, and $\mathrm{Pd}$ prefer that geometry after 300 atoms, while $\mathrm{Cu}$ favours this morphology above 1000 atoms [28]. We, thus, conclude that soft inter-particle potentials elements, such as copper, not only show a preference for Ih structures in a larger size range but also favour concerted motions towards this morphological basin, opposite to elements characterized by a more stiff interaction, as in the case of gold.

It is commonly observed that the last size at which MetaD sample concerted mechanisms is larger than the itMD one. Indeed, MetaD enhances the sampling of structures at fixed temperature, while itMD simulations can hide possible structural transition mechanisms if the temperature is rescaled at a too quick pace, hinting to the competition of the DSD rearrangements with surface diffusion driven ones for sizes close to the critical one.

\section{Conclusion}

By systematically assessing structural transitions in noble and quasi-noble metals of $0.5-2.0 \mathrm{~nm}$ diameter, we identify numerous structural rearrangements and characterize them according to the evolution of their nearest neighbours network. We unveil a clear size and material dependence of the probability to observe a concerted rearrangement mechanism at ambient temperature. Concerted motions are favourable only up to a critical size above which continuous surface diffusion driven rearrangement mechanisms and defects formation are more likely to take place. The former lead to a different structural basin, the latter do not.

We remark that the kind and number of nanoparticle's active sites may be strongly affected by solid-solid rearrangements, hence leading to a significant modification of their catalytic performance. Being able to detail guidelines about the possible changes of metastable geometries may thus be of great help in predicting and rationalizing the catalytic properties of metallic nanoparticles at finite-temperature.

FB and LP thank the financial support by the U.K. Engineering and Physical Sciences Research Council (EPSRC), under Grant Nos. EP/GO03146/1 and EP/J010812/1. KR acknowledges financial support by EPSRC, Grant No. ER/M506357/1 and by the Thomas Young Center Junior Research Fellowship. YYS benefits of a MyBrainSc scholarship sponsored by the Malaysian government. The authors thank the financial support offered by Royal Society under the project number RG120207.

\section{Author contribution statement}

KR and YS performed the numerical calculations; LP, KR and FB co-authored the numerical code. KR and FB analyzed the data and wrote the manuscript. FB had the original idea of the manuscript.

Open Access This is an open access article distributed under the terms of the Creative Commons Attribution License (http://creativecommons.org/licenses/by/4.0), which permits unrestricted use, distribution, and reproduction in any medium, provided the original work is properly cited.

\section{References}

1. F. Baletto, R. Ferrando, Phys. Chem. Chem. Phys. 17, 27920 (2015)

2. F. Calle-Vallejo, J. Tymoczko, V. Colic, Q.H. Vu, M.D. Pohl, K. Morgenstern, D. Loffreda, P. Sautet, W. Schuhmann, A.S. Bandarenka, Science 350, 185 (2015)

3. G.G. Asara, L.O. Paz-Borbón, F. Baletto, ACS Catal. 6, 4388 (2016)

4. C. Di Paola, R. D'Agosta, F. Baletto, Nano Lett. 16, 2885 (2016)

5. P.L. Hansen, J.B. Wagner, S. Helveg, J.R. Rostrup-Nielsen, B.S. Clausen, H. Topsøe, Science 295, $5562(2002)$

6. M.A. Newton, C. Belver-Coldeira, A. Martínez-Arias, M. Fernández-García, Nat. Mater. 6, 528 (2007)

7. W. Xu, J.S. Kong, Y.-T.E. Yeh, P. Chen, Nat. Mater. 7, $992(2008)$

8. K. Koga, T. Ikeshoji, K.-I. Sugawara, Phys. Rev. Lett. 92, 115507 (2004)

9. Z.W. Wang, R.E. Palmer, Phys. Rev. Lett. 108, 245502 (2012)

10. S.R. Plant, L. Cao, R.E. Palmer, J. Am. Chem. Soc. 136, 7559 (2014)

11. A.L. Mackay, Acta Crystallogr. 15, 916 (1962)

12. W.N. Lipscomb, Science 153, 373 (1966)

13. D.J. Wales, L.J. Munro, J. Phys. Chem. 100, 2053 (1996)

14. T.X. Li, S.M. Lee, S.J. Han, G.H. Wang, Phys. Lett. A, 300, 86 (2002)

15. Z. Zhang, W. Hu, S. Xiao, Phys. Rev. B 73, 125443 (2006)

16. F. Chen, R.L. Johnston, Appl. Phys. Lett. 92, 023112 (2008)

17. B. Cheng, A.H.W. Ngan, J. Chem. Phys. 138, 164314 (2013) 
18. Y.K. Lan, C.H. Su, W.H. Sun, A.C. Su, RSC Adv. 4, 13768 (2014)

19. A.L. Gould, A.J. Logsdail, C.R.A. Catlow, J. Phys. Chem. C 119, 23685 (2015)

20. L. Pavan, K. Rossi, F. Baletto, J. Chem. Phys. 143, 184304 (2015)

21. A.L. Gould, K. Rossi, C.R.A. Catlow, F. Baletto, A.J. Logsdail, J. Phys. Chem. Lett. 7, 4414 (2016)

22. K. Rossi, F. Baletto, Phys. Chem. Chem. Phys. 19, 11057 (2017)

23. K. Rossi, T. Ellaby, L.O. Paz-Borbón, I. Atanasov, L. Pavan, F. Baletto, J. Phys: Condens. Matter 29, 145402 (2017)

24. S. Trygubenko, D. Wales, J. Chem. Phys. 120, 2082 (2004)
25. F. Baletto, R. Ferrando, Surf. Sci. 490, 361 (2001)

26. F. Baletto, C. Mottet, R. Ferrando, Surf. Sci. 446, 31 (2000)

27. S.Y. Kim, I.-H. Lee, S. Jun, Phys. Rev. B, 76, 245407 (2007)

28. F. Baletto, R. Ferrando, A. Fortunelli, F. Montalenti, C. Mottet, J. Chem. Phys. 116, 3856 (2002)

29. J.D. Honeycutt, H.C. Andersen, J. Phys. Chem. 91, 4950 (1987)

30. V. Rosato, M. Guillope, B. Legrand, Philos. Mag. A 59, 321 (1989)

31. A. Laio, F.L. Gervasio, Rep. Progr. Phys. 71, 126601 (2008)

32. E. Apra, F. Baletto, R. Ferrando, A. Fortunelli, Phys. Rev. Lett. 93, 065502 (2004) 


\section{Appendix A}

Table A.1 summarizes the characterization of the structural transitions observed when starting from a Co geometry, as done in the case of the initial $\mathrm{Dh}$ architecture discussed in the main text. Mechanism crossover sizes, characteristic transition temperatures and $\overline{C^{m a x}}$ values display a qualitatively similar behaviour to the data discussed for the case of the Dh. The label dFCC refers to a defected FCC morphological basin.

Table A.1. Per each system (leftmost column) we report $\rightarrow \operatorname{Ih} \%, \overline{C_{\rightarrow I h}^{\max }}$ and $\overline{T_{\rightarrow I h}}$, or, $\overline{C_{T \rightarrow d F C C}^{\max }}$ and $\overline{T_{\rightarrow d F C C}}$, for

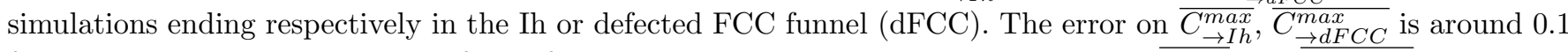
for the concertedness index and of 0.03 for the characteristic transition temperatures $\overline{T_{\rightarrow I h}}$ and $\overline{T_{\rightarrow d F C C}}$. Rightmost column reports $\overline{\Delta F_{\rightarrow I h}}$, and $\overline{\Delta F_{\rightarrow F C C}}$ in bracket if observed. Error on $\overline{\Delta F_{\rightarrow I h}}\left(\overline{\Delta F_{\rightarrow F C C}}\right)$ is of the order of the height of one Gaussian. $\overline{\Delta F \rightarrow I h^{*}}$ instead represents an upper bound of the true free energy barrier.

\begin{tabular}{|c|c|c|c|c|c|c|}
\hline System & $\rightarrow \mathrm{Ih} \%$ & $\overline{C_{\rightarrow I h}^{\max }}$ & $\overline{T_{\rightarrow I h}}$ & $\overline{C_{\rightarrow d F C C}^{\max }}$ & $\overline{T_{\rightarrow d F C C}}$ & $\overline{\Delta F_{\rightarrow I h}^{(*)}}\left(\overline{\Delta F_{\rightarrow F C C}}\right)(\mathrm{eV})$ \\
\hline $\mathrm{Ni}_{55}$ & 100 & 1.0 & $<0.02$ & & & $<0.05$ \\
\hline $\mathrm{Ni}_{147}$ & 100 & 0.9 & 0.38 & & & $0.1(3.5)$ \\
\hline $\mathrm{Ni}_{309}$ & 62 & 0.6 & 0.62 & 0.2 & 0.34 & 0.3 \\
\hline $\mathrm{Ni}_{561}$ & 0 & & & 0.2 & 0.45 & 0.7 \\
\hline $\mathrm{Pd}_{55}$ & 100 & 1.0 & 0.23 & & & 0.05 \\
\hline $\mathrm{Pd}_{147}$ & 100 & 0.9 & 0.60 & & & $0.5(3.0)$ \\
\hline $\mathrm{Pd}_{309}$ & 0 & & & 0.3 & 0.58 & 3.7 \\
\hline $\mathrm{Pd}_{561}$ & 0 & & & 0.2 & 0.48 & \\
\hline $\mathrm{Pt}_{55}$ & 100 & 1.0 & 0.39 & & & 0.25 \\
\hline $\mathrm{Pt}_{147}$ & 100 & 0.8 & 0.55 & & & 1.7 \\
\hline $\mathrm{Pt}_{309}$ & 0 & & & 0.3 & 0.49 & \\
\hline $\mathrm{Pt}_{561}$ & 0 & & & 0.2 & 0.42 & \\
\hline $\mathrm{Cu}_{55}$ & 100 & 1.0 & $<0.02$ & & & $<0.05$ \\
\hline $\mathrm{Cu}_{147}$ & 100 & 1.0 & $<0.02$ & & & 0.1 \\
\hline $\mathrm{Cu}_{309}$ & 100 & 1.0 & $<0.02$ & & & 0.3 \\
\hline $\mathrm{Cu}_{561}$ & 100 & 0.8 & 0.33 & & & 0.7 \\
\hline $\mathrm{Ag}_{55}$ & 100 & 0.9 & $<0.02$ & & & $<0.05$ \\
\hline $\operatorname{Ag}_{147}$ & 100 & 0.8 & 0.47 & & & $0.4(3.2)$ \\
\hline $\mathrm{Ag}_{309}$ & 0 & & & 0.3 & 0.57 & 2.5 \\
\hline $\mathrm{Ag}_{561}$ & 0 & & & 0.2 & 0.51 & \\
\hline $\mathrm{Au}_{55}$ & 100 & 0.9 & 0.40 & & & $<0.05$ \\
\hline $\mathrm{Au}_{147}$ & 0 & & & 0.3 & 0.61 & 0.5 \\
\hline $\mathrm{Au}_{309}$ & 0 & & & 0.2 & 0.50 & \\
\hline $\mathrm{Au}_{561}$ & 0 & & & 0.1 & 0.41 & \\
\hline
\end{tabular}

